\title{
Validation of the Thai version of the family reported outcome measure (FROM-16)@ to assess the impact of disease on the partner or family members of patients with cancer
}

\author{
Pattariya Chantarasap ${ }^{1}$, Nutjaree Pratheepawanit Johns ${ }^{2}$, Srivieng Pairojkul ${ }^{3}$, Aumkhae Sookprasert ${ }^{4}$,
} Kosin Wirasorn ${ }^{4}$, Areewan Cheawchanwattana ${ }^{5}$, Sam Salek ${ }^{6}$ and Suphat Subongkot ${ }^{7 *}$ (D)

\begin{abstract}
Background: Cancer not only impairs a patient's physical and psychosocial functional behaviour, but also contributes to negative impact on family members' health related quality of life. Currently, there is an absence of a relevant tool in Thai with which to measure such impact. The aim of this study was to translate and validate the Family Reported Outcome Measure (FROM-16) in Thai cancer patients' family members.

Methods: Thai version of FROM-16 was generated by interactive forward-backward translation process following standard guidelines. This was tested for psychometric properties including reliability and validity, namely content validity, concurrent validity, known group validity, internal consistency, exploratory and confirmatory factor analysis. Construct validity was examined by comparing the Thai FROM-16 version with the WHOQOL-BREF-THAI.

Results: The internal consistency reliability was strong (Cronbach's alpha $=0.86)$. A Negative moderate correlation between the Thai FROM-16 and WHOQOL-BREF-THAI was observed $(r=-0.4545, p<0.00)$, and known group validity was proved by a statistically significant higher score in family members with high burden of care and insufficient income. The factor analysis supported both 3-factor and 2-factor loading model with slight difference when compared with the original version.

Conclusions: The Thai FROM-16 showed good reliability and validity in Thai family members of patients with cancer. A slight difference in factor analysis results compared to the original version could be due to cross-culture application.
\end{abstract}

\section{Background}

Health related quality of life (HRQOL) is of importance and considered an ultimate treatment goal for many conditions. HRQOL assessment has been widely used not only in research but also in clinical practice [1]. It is especially valuable in chronic diseases such as cancer, where patients may suffer for a long duration from the disease condition, complications or even the treatment process [1]. Although healthcare providers have turned

\footnotetext{
* Correspondence: supsub2@kku.ac.th; supsub2@gmail.com

The abstract was presented at the 2017 ESMO Asia Congress, Nov 18 $8^{\text {th }}, 2017$, Singapore.(Abstract Number: 478P)

${ }^{7}$ Division of Clinical Pharmacy, Faculty of Pharmaceutical Sciences, Khon Kaen University, Khon Kaen, Thailand

Full list of author information is available at the end of the article
}

their attention to patient's HRQOL, the family's HRQOL is usually overlooked. There is evidence that suggests a negative impact on family members in term of caregiving burden, finance, life style, or even the mental status $[2,3]$. Study also shows that most family members needs are not met by healthcare services, resulting in distress and anxiety [4].

More importantly, a patient's HRQOL may depend significantly on the HRQOL of the family members. The impact on quality of life for people who have family members suffering from cancer are expected to have circumstance that differ from the normal population $[5,6]$. This is more profound in Eastern culture and Asian families who tend to be more holistic and interdependent, resulting in

(c) The Author(s). 2019 Open Access This article is distributed under the terms of the Creative Commons Attribution 4.0 International License (http://creativecommons.org/licenses/by/4.0/), which permits unrestricted use, distribution, and reproduction in any medium, provided you give appropriate credit to the original author(s) and the source, provide a link to the Creative Commons license, and indicate if changes were made. The Creative Commons Public Domain Dedication waiver (http://creativecommons.org/publicdomain/zero/1.0/) applies to the data made available in this article, unless otherwise stated. 
different cognitive and social orientation when compared with Western populations [7]. There is a lack of relevant instruments to apply in clinical settings [8] with only the recently reported Family Reported Outcome Measure (FROM-16), containing 16 items developed for family members of chronic disease patients in United Kingdom, being available. It has good psychometric properties and able to be used in routine practice taking only two minutes for questionnaire completion time [9]. The study aimed at translating and validating a Thai version of FROM-16 in Thai family members of cancer patients.

\section{Method}

\section{Ethical approval}

The study protocol was approved by the Khon Kaen University Ethics Committee in Human Research. Written informed consent was obtained from both family members and patients prior to data collection.

\section{Study participants Family members}

Family members who were eligible for the study were approached at both outpatient and inpatient units at Srinagarind Hospital, a public tertiary care university teaching facility. Only one family member was recruited for each patient. The main recruitment setting was at a chemotherapy administration unit, with few participants also recruited from radiation and surgical units. The inclusion criteria for eligibility were:

- a family member of a patient who was diagnosed with cancer,

- 18 years of age or older

- able to read and write in Thai.

- able to give written informed consent

- attending the hospital as inpatients or outpatients

\section{Patients}

Patients were included if they are 18 years old of age or older, had any solid neoplasm or myeloid or lymphoid neoplasm or acute leukemia according to 2008 WHO classification (2008) [10] at any stage of disease diagnosis, during 1st line treatment, treatment failure and/or resistance, 2nd line and subsequent treatments. Furthermore, patients had to also satisfy the following inclusion criteria:

\section{Translation, content validity and practicality}

Thai FROM-16 was generated by an interactive forwardbackward translation process [11]. Two independent translators were assigned for forward translation. The reconciled version of the questionnaire was sent to five experts for reviewing and suggestions. Cognitive debrief interview of the reconciled forward translation was conducted with five family members. The understanding and clarity of the questions as well as issues concerning the appropriateness of questionnaire were investigated. The revised forward translation was then back translated by another two independent translators for final review.

\section{Procedure and measures}

The participants were asked to complete Thai FROM-16 along with validated World Health Organization Quality of Life Brief-Thai (WHOQOL-BREF-THAI). All were done by self-administration in alternate order of the questionnaire. Time to complete the Thai FROM-16, and demographic data of the family member were recorded.

As the original version, Thai FROM-16 measure contains 16 items relating with impact of patient illness on family members. Two concepts of domain were identified in original version, including 'emotional' (items 1-6) and 'personal and social life' (items 7-16). The frame of response is "at this moment" with three response options, scoring from 0 to 2 when higher score indicates more impact. Scores could be calculated with total score of maximum 32 or each domain with 6 items in domain 1 (maximum score 12) and 10 items in domain 2 (maximum score 20). Ceiling and floor effect were also obtained in each domain and total score.

WHOQOL-BREF-THAI is a 26-item measure that has been officially used by Thai Department of Mental Health, Ministry of Public Health, for quality of life measures. It shows good psychometric properties in the Thai population [13]. Four domains measured by WHOQOL-BREF-THAI are physical domain, psychological domain, social relationship, environment, and two overall health scores. The answers consist of five response options, scoring from 26 to 130 where a lower score indicates worst HRQOL.

All statistical analysis was performed using STATA version 12.0, except for confirmatory factor analysis (CFA), which were done by LISREL version 9.20. Content validity was performed during the translation process by expert panel, pre-test and cognitive interviewing. Known group validity was performed based on the hypothesis that the HRQOL of family members who were female, family of a patient who was diagnosed with advanced disease (metastasis stage, relapsed/refractory, and acute leukemia), had insufficient income, were full time caregiving, and of whom the patient was unable to take care of daily living would be more affected $[6,13]$. Statistical analysis was tested using Mann-Whitney $\mathrm{U}$ test, and $p$ value $<0.05$ was considered statistically significant. Concurrent, convergent and divergent validity were performed using Spearman's correlation between Thai FROM-16 and WHOQOLBREF-THAI based on 2 hypothesizes. First, both Thai FROM-16 total and domain score should be negatively correlated with WHOQOL-BREF-THAI total score to 
reflect the impact of patient condition on family member HRQOL as reported in the original English version [9]. Second, the emotional domain of Thai FROM-16 should be negatively correlated with the psychological domain but not physical health, social relationship, and environment domains of WHOQOL-BREF-THAI. Correlation coefficients of 0.0 to $0.3,0.3$ to $0.5,0.5$ to $0.7,0.7$ to 1.0 were considered to show negligible, low, moderate, and strong association, respectively [14]

Exploratory factor analysis (EFA) and confirmatory factor analysis (CFA) were applied to determine factor loading and compare with the original English version. Selected goodness of fit parameters for CFA consist of non-significant Chi Square test $\left(X^{2}\right)$, Root Mean Square Error of Approximation (RMSEA) less than 0.6, Comparative Fit Index (CFI), Normed Fit Index (NFI), Non-Normed Fit Index (NNFI) with range 0 (no fit) to 1 (perfect fit), and Standardized RMR (SRMR) less than 0.05 being considered as a good fit [15]. /the reliability test was performed by using internal consistency with expected Cronbach's alpha $\geqq 0.7$ being considered as an acceptable value $[16,17]$.

\section{Result}

\section{Family members and patient}

Two hundred and fifty family members were recruited to the study between February and December 2016, from both oncologic (71.38\%) and hematologic malignancy (28.62\%) specialty. Two participants were excluded due to uninterpretable of WHOQOL-BREF-THAI score, considering $>0.8 \%$ missing data. Two hundred and forty-eight were included for final data analysis. Most of patients were diagnosed with advanced or metastatic stage cancer (50\%), with median duration of disease of four months (range 196), and currently receiving chemotherapy for the treatment (97\%) (Table 1). The majority of family members were female $(70 \%)$ with mean age of 44 years, ranging from 18 to 70 . They were mostly married (74.19\%), had education level of bachelor's degree or higher (41.53\%), and being either spouse (41\%) or child (40\%) of the patient (Table 2.)

\section{Thai FROM-16 score}

The score results are shown in Table 3. Score of zero was observed in $1(0.4 \%)$ in the emotional domain and 4 $(1.6 \%)$ in the personal and social life domain. Maximum score was only observed in $2(0.8 \%)$ in the emotional domain. Using Spearman's rank correlation coefficient, there are strong positive correlations between both domains to the total score, $r=0.81, p<0.001$, and 0.94 , $p<0.001$, respectively. The two domains also show moderate correlation to each other, $r=0.59, p<0.001$. The highest mean score was observed in item 15: increase cost, followed by item 1: feeling worry, item 3: feeling
Table 1 Patient disease, staging and treatment $(N=248)$

\begin{tabular}{ll}
\hline Diagnosis number of patient (\%) & \\
Lymphoma 43 (17.7) & Gastric cancer 9 (3.6) \\
Hepatobiliary cancer 38 (15.3) & Soft tissue sarcoma 9 (3.6) \\
Lung cancer 27 (10.9) & Bone cancer 8 (3.2) \\
Colon \& rectal cancer 27 (10.9) & Bladder cancer 4 (1.5) \\
Head and neck cancer 21 (8.4) & Prostate cancer 3 (1.2) \\
Breast cancer 20 (8) & Testicular cancer 2 (0.8) \\
Multiple myeloma 17 (6.9) & Esophageal cancer 2 (0.8) \\
Leukemia 10 (4) & Other 8 (3.2) \\
Stage of disease (\%) & \\
Stage 1 & $6(2.4)$ \\
Stage 2 & $26(10.1)$ \\
Stage 3 & $45(18.2)$ \\
Stage 4 & $123(49.6)$ \\
Relapse & $38(15.3)$ \\
Not relevant & $10(4.4)$ \\
Treatment (\%) & \\
Chemotherapy & $241(97.2)$ \\
CCRT & $5(2.0)$ \\
Surgery & $1(0.4)$ \\
Radiation & $1(0.4)$ \\
\hline
\end{tabular}

${ }^{a}$ Other includes ovary, uterus, anal, CNS, pancreas, peritoneal, and unknown primary cancer

CRRT Concurrent Chemotherapy and radiation

sad, and item 16: affected sleep. The lowest mean scores were observed in item 2: feeling angry and item 12: sex life. Neither age nor duration of disease achieved a significant correlation level with the Thai FROM-16 score.

\section{Content validity and practicality}

Pre-test and cognitive interviewing of the final version then were done in five family members. The results showed that items of Thai FROM-16 were easy to understand of $96 \%$ of the study subjects. Two family members stated that item 6 and item 12 could be interpreted differently by others, but they were able to understand the intention of the questions, hence, no change was made at this stage. All family members agreed that the response options were clear, and no new questions were suggested. Two family members were excluded from time analysis due to an issue of behavior during administration, which resulted in difficulty of time measurement. The questionnaire was completed within three minutes with median time of $160 \mathrm{~s}$ (range $51-520 \mathrm{~s}, n=246$ ). A significant difference in median time of administration was observed between education group with a median of $201 \mathrm{~s}$ in the under high school group versus $160 \mathrm{~s}$ in high school or over group, $p<0.001$. 
Table 2 Demographic characteristics of the study participants $(N=248)$

\begin{tabular}{|c|c|}
\hline & Value (\%) \\
\hline \multicolumn{2}{|l|}{ Family members } \\
\hline \multicolumn{2}{|l|}{ Gender, $n(\%)$} \\
\hline Male & $74(29.8)$ \\
\hline Female & $174(70.2)$ \\
\hline \multicolumn{2}{|l|}{ Age (years) } \\
\hline Mean & 44 \\
\hline Median & 44 \\
\hline Range & $18-70$ \\
\hline \multicolumn{2}{|l|}{ Martial Status } \\
\hline Married & $184(74.2)$ \\
\hline Single & $52(21.0)$ \\
\hline Divorced/Widowed & $12(4.8)$ \\
\hline \multicolumn{2}{|l|}{ Relationship to patient, n (\%) } \\
\hline Spouse/partner & $101(40.7)$ \\
\hline Child & $98(39.5)$ \\
\hline Sibling & $19(7.8)$ \\
\hline Parent & $15(6)$ \\
\hline Other $^{a}$ & $15(6)$ \\
\hline \multicolumn{2}{|l|}{ Education, $n(\%)$} \\
\hline Bachelor's degree or higher & $103(41.5)$ \\
\hline Vocational certificate & $15(6.1)$ \\
\hline High school & $68(27.4)$ \\
\hline Under high school & $62(25)$ \\
\hline \multicolumn{2}{|l|}{ Patient } \\
\hline \multicolumn{2}{|l|}{ Gender, $n(\%)$} \\
\hline Male & $138(55.4)$ \\
\hline Female & $110(44.6)$ \\
\hline \multicolumn{2}{|l|}{ Age (years) } \\
\hline Mean & 53 \\
\hline Median & 56 \\
\hline Range & $18-76$ \\
\hline \multicolumn{2}{|l|}{ Duration of disease (months) } \\
\hline Mean & 6 \\
\hline Median & 4 \\
\hline Range & $1-96$ \\
\hline \multicolumn{2}{|l|}{ Coverage/payment } \\
\hline Universal coverage & $123(49.6)$ \\
\hline Government/state enterprise officer & $103(41.5)$ \\
\hline Social security scheme & $14(5.7)$ \\
\hline other $^{\mathrm{b}}$ & $8(3.2)$ \\
\hline
\end{tabular}

Includes grandchild, uncle/aunt, grandparent, cousin

${ }^{b}$ self-pay, private insurance
Concurrent validity and convergent and divergent validity The results show a negative correlation between the total score of Thai FROM-16 and WHOQOL-BREF-THAI ( $r=$ $-0.4545, p<0.001)$. Similar results were found in both the emotional domain $(r=-0.3, p<0.001)$ and personal and social life domain $(r=-0.4624 ; p<0.001)$. A low negative correlation was observed between emotional and psychological domain $(r=-0.3118, p<0.001)$, however, by contrast, negligible correlation was found between the emotional domain and physical health $(r=-0.2502, p<$ $0.001)$, social relationship $(r=-0.2158, p<0.001)$, and environment $(r=-0.1869, p<0.001)$, respectively.

\section{Factor analysis}

Exploratory factor analysis (EFA) was performed to identify factor loading in Thai FROM-16. Principal component analysis was used as the extraction method, and factors with eigenvalues $\geqq 1$ according to Kaiser's criteria rule and Cattells' scree plot were retained for orthogonal oblimin rotation with Kaiser normalisation $[18,19]$. Two factors loading with items 1-6 and 7-16 were expected. Two hundred and forty-eight subjects were included in the EFA, which produced 3 factors with eigenvalues $\geqq 1$, explaining $47.73 \%$ of the variance. One of the retained factors was very close to lower limit cut-off point with eigenvalues of 1.09 and scree plot showed two potential factors as reported in the original version. The 3-factor, 2-factor and 1-factor models were retained for rotation to identified factor loading in each item. All items achieved the minimum value of 0.4 , which considered as a significant level [19]. (Table 4) All three models resulted in different item loadings when compared with the original model.

In order to measure fitness of model fit, confirmatory factor analysis (CFA) was applied, including the original questionnaire for comparison as shown in Table 5. All of the models showed similar goodness of fit indices with slightly better values in the 3-Factor model. The chi-squared test is statistically significant in all models, which may result from the large sample size [20]. However, none of the models resulted in a very good fit level.

\section{Known group validity}

A known group analysis was performed for the validity test. The median of Thai FROM-16 total score and WHOQOL-BREF-THAI total score were compared between groups as show in Table 6. The result shows a statistically higher FROM-16 score in total and all domains for family member with insufficient income when compared with the sufficient income group. A similar result also shows in total score but not individual domains for family members who stated themselves as the full time caregiver versus part time or non-caregiver group. The statistically significant higher score was revealed in total 
Table 3 Thai FROM16 score data $(N=248)$

\begin{tabular}{lllllll}
\hline Domain & Mean & SD & Median & Range & Floor (\%) & Ceiling (\%) \\
\hline Total score (0-32) & 11.75 & 5.85 & 11 & $1-31$ & - & - \\
Emotional domain score (0-12) & 4.7 & 2.46 & 4 & $0-12$ & 0.4 & 0.8 \\
Personal and social life domain (0-20) & 7.1 & 3.4 & 7 & $0-19$ & 1.6 & - \\
\hline
\end{tabular}

and 'personal and social life' domain for family members who live with impaired physical function patient. There was no statistically significant difference between male and female. The same finding was found in the disease severity group. The lower score for WHOQOLBREF-THAI was also observed in the insufficient income and full time care giver groups with score of 92 versus $102(p<0.001)$ and 93 versus 96, $(p=0.044)$, respectively. However, the score was not significantly different according to sex, severity of disease, and patient's physical function status.

\section{Reliability}

The overall Thai FROM-16 and each domain achieved good values for Cronbach's alpha with 0.86 for total score, 0.73 for domain 1 (emotional) and 0.82 for domain 2 (personal and social life). Alphas if Items were deleted were also measured with no significant improvement of alpha by deleting each item $(0.85-0.86)$. Similar results were shown in each domain with a range of alpha if Item deleted from $0.67-0.72$ and $0.79-0.82$ for domain 1 and domain 2 , respectively.

\section{Discussion}

The findings show that the Thai version of FROM 16 is valid and reliable in Thai family members of cancer patients, with slightly different factor loading structure to the original version. Short time of administration was also observed with median time approximately $3 \mathrm{~min}$ (160 s).

FROM-16 is a questionnaire originated in English language. Validation tests reflect good psychometric properties in family member of chronic illness patient in the United Kingdom. The questionnaire is also highly applicable for routine use due to simplicity and short administration time. The affected area that is covered by FROM-16 was relevant to the most identified impact on Thai family members [8], but cross-cultural adaptation of questionnaire in population with different language may not feasible unless an appropriate translation process and psychometric properties tests were confirmed [21].

Neither floor nor ceiling effect was observed in either total or domain score analysis, with $15 \%$ being considered the maximum acceptable [22]. This indicates that the Thai FROM-16 is suitable for measuring the impact

Table 4 Result of exploratory factor analysis: the factor loading matrix of Thai FROM16 and original version ${ }^{10}$

\begin{tabular}{|c|c|c|c|c|c|c|}
\hline \multirow{2}{*}{$\begin{array}{l}\text { Item number and description } \\
1 . \text { I feel worried }\end{array}$} & \multicolumn{3}{|c|}{ 3-Factor Model } & \multicolumn{2}{|c|}{ 2-Factor Model } & \multirow{2}{*}{$\frac{1-\text { Factor Model }}{0.532}$} \\
\hline & 0.392 & 0.115 & 0.679 & 0.436 & 0.659 & \\
\hline 2 I feel angry & 0.332 & 0.618 & 0.135 & 0.504 & 0.069 & 0.509 \\
\hline 3 I feel sad & 0.411 & 0.195 & 0.705 & 0.479 & 0.677 & 0.576 \\
\hline 4 I feel frustrated & 0.470 & 0.479 & 0.239 & 0.603 & 0.184 & 0.624 \\
\hline $5 \mathrm{It}$ is difficult to find someone to talk to about my thought & 0.474 & 0.597 & -0.037 & 0.509 & -0.100 & 0.488 \\
\hline 6 Caring for my family member is difficult & 0.501 & 0.438 & -0.022 & 0.606 & -0.072 & 0.588 \\
\hline 7 It is hard to find time for myself & 0.573 & 0.139 & -0.225 & 0.578 & -0.245 & 0.535 \\
\hline 8 My every day travel is affected & 0.620 & 0.034 & 0.161 & 0.609 & 0.149 & 0.624 \\
\hline 9 My eating habits are affected & 0.607 & 0.164 & -0.004 & 0.627 & -0.028 & 0.616 \\
\hline 10 My family activities are affected & 0.670 & -0.174 & 0.199 & 0.597 & 0.207 & 0.622 \\
\hline 11 I experience problem with going on holiday & 0.525 & 0.186 & -0.184 & 0.548 & -0.208 & 0.511 \\
\hline 12 My sex life is affected & 0.449 & 0.175 & -0.399 & 0.464 & -0.420 & 0.395 \\
\hline 13 My work or study is affected & 0.697 & -0.227 & 0.017 & 0.600 & 0.031 & 0.598 \\
\hline 14 My relationship with other family members are affected & 0.695 & 0.059 & 0.052 & 0.683 & 0.038 & 0.681 \\
\hline 15 My family expense are & 0.591 & 0.037 & 0.049 & 0.577 & 0.038 & 0.576 \\
\hline 16 My sleep is affected & 0.686 & -0.087 & 0.206 & 0.638 & 0.206 & 0.662 \\
\hline
\end{tabular}


Table 5 Comparison of fit indices between models

\begin{tabular}{llll}
\hline Goodness-of-Fit Statistics & 3-Factor Model & 2-Factor Model & 1-Factor Model \\
\hline Degrees of Freedom & 87 & 89 & 104 \\
Chi-Square (C1) & 278.2955 & 323.2056 & 487.8074 \\
& $(P<0.001)$ & $(P<0.001)$ & $0.0000)$ \\
Root Mean Square Error of Approximation (RMSEA) & 0.0941 & 0.1030 & 0.122 \\
Comparative Fit Index (CFI) & 0.8701 & 0.8440 & 0.7736 \\
Normed Fit Index (NFI) & 0.8244 & 0.7988 & 0.7312 \\
Non-Normed Fit Index (NNFI) & 0.8432 & 0.8160 & 0.7387 \\
Standardized RMR (SRMR) & 0.0575 & 0.0617 & 0.0716 \\
\hline
\end{tabular}

in this population. The mean and median scores were very similar to the original study [9], but tended to be lower in the cancer-related population. This finding may result from two reasons; the sampling method and cultural issues. The purposive sampling method by picking up the small group of most represented subjects, assessed by senior specialist in each specialty may result in higher scores when compare with a larger sample size. Second, the culture context in terms of caring for Thai family members, according to the finding in the interactive review data, considers taking care of a patient in the family as a gratitude, commitment, and responsibility [8], hence may result in lower response as an impact or burden. Furthermore, sex life is considered as a sensitive issue in Asian populations and may limit the response. No correlation was found with age and disease duration. This finding needs to be further confirmed in multivariate analysis before conclusions could be drawn.

The content validity was performed by a multidisciplinary expert panel, including physicians, pharmacists, and a nurse. Cognitive and debriefing results of the final version shows that Thai FROM-16 is easy to understand from both question and response options perspectives. During the pretest, there was no change for any items suggested by family members. The concurrent validity was confirmed by negative correlation between Thai FROM-16 and WHOQOL-BREF-THAI, indicating that higher impacts may contribute to lower HRQOL. Convergent and divergent validity, tested with hypothesis of negligible correlation for unrelated domains, also indicates a good result. On the other hand, this test score showed expected negative correlation with psychological domain in WHOQOL-BREF-THAI. Known group validity was confirmed based on literature findings of negative effect on certain groups of family members' HRQOL. Female family member, having a higher burden as a caregiver, living with patient with advanced disease or limited physical ability of patients and those who have insufficient income were expected to be more affected [12]. These tests also reflected positive result for the hypothesis in terms of care and financial burden, with statistically significant higher Thai FROM-16 total scores. In contrast, there was no difference according to sex and severity of disease.

An interesting issue was found in factor analysis results. Three factors that exceeded the eigenvalue cutoff point were identified by PCF extraction and Cattells' scree plot, which very similar to the original version, one eigenvalue was close to lower limit of 1 and may be consider to be removed. However, the oblimin rotation reveals different factor loading for both models, as seen in the structure matrix, where items 4 and 6 significantly loaded on two components with very close values. In order to clarify these issues, CFA was used to identify the best fit. All factor loading models including the original one were included for analysis. The 3-factor model revealed slightly better values for selected goodness of fit indices. This model consists of component one: item 1 and 3, component two: item 2, 4, 5, and 6, component three: items 7-16. The last component is similar to original version, while the emotional part was divided into 2 components. This finding may result from difference cultural aspects of Asian and Western populations. According to the cognitive interview in the pilot study,

Table 6 Known group validity $(N=248)$

\begin{tabular}{lllll}
\hline Group (N) & $\begin{array}{l}\text { FROM16 total } \\
\text { score }\end{array}$ & $\begin{array}{l}\text { Emotional domain } \\
\text { score }\end{array}$ & $\begin{array}{l}\text { Personal and social } \\
\text { life domain score }\end{array}$ & $\begin{array}{l}\text { WHOQOL-BREF- } \\
\text { Score }\end{array}$ \\
\hline Female (174) VS Male (74) & 12 VS 10 $p=0.8304$ & 4.5 VS 40.5351 & 7 VS $7 p=0.9011$ & 93 VS $93 p=0.8141$ \\
Advanced disease(171) VS Early stage (77) & 12 VS 10 $p=0.4606$ & 4 VS $4 p=0.7357$ & 7 VS $6 p=0.3941$ & 93 VS $94 p=0.9063$ \\
Sufficient income (62) VS Insufficient income (186) & 12 VS $8 p<0.001$ & 5 VS $3 p=0.003$ & 7 VS $5 p<0.001$ & 92 VS $102 p<0.001$ \\
Full time care giver (158) VS Part time/Not a care giver (90) & 12 VS 10 $p=0.034$ & 5 VS $4 p=0.2$ & 7 VS $6 p=0.528$ & 93 VS $96 p=0.044$ \\
ADL dependent patient (50) VS independent patient (198) & 13 VS 11 $p=0.034$ & 5 VS $4 p=0.055$ & 8 VS $6.5 p=0.043$ & 92 VS $94.5 p=0.0987$ \\
\hline
\end{tabular}


item 1: feeling worry and item 3: feeling sad were considered as self-emotion, and could keep it by themself, while item 2: feeling angry, item 4: feeling frustrated, item 5: difficult to find someone to talk to, and item 6: feeling difficulty of caring, were considered as the feeling that may affect the patient and/or other family members. This finding is consistent with the pattern of structural difference in cross-cultural adaptations in both generic and specific instrument in Asian countries [22, 23, 24].

A Cronbach's alpha value of 0.86 reflects a good reliability of Thai FROM-16. The two domains also archived an acceptable value by Cronbach's alpha of 0.73 and 0.82 . Possibility of item drop out was demonstrated by alpha if item deleted analysis. None of removed items resulted in significant improvement of alpha values, indicating that all items contributed to the whole questionnaire and none should not be deleted.

This study had several limitations. All participants were recruited from a single center and most of the patients were receiving chemotherapy as the treatment. However, Srinagarind Hospital is the main tertiary referral center providing medical services across the northeast region of Thailand. Our sample was diagnosed with both oncologic and hematologic malignacy, and mostly diagnosed in advanced stages and currently receiving chemotherapy, hence these family members should be a good representative for the Thai population. Furthermore, chemotherapy is known as a treatment that contribute to the negative effects on HRQOL for both cancer patient and family members, [25] and will be a good reflection for negative impact in a cancer population.

\section{Conclusion}

This study shows good reliability and validity of the Thai version of FROM-16 with some differences in structural factor loadings. The questionnaire also requires short time for administration, so is suitable for routine clinical use. These findings generally support using of this questionnaire in Thai family members of cancer patients. The future challenge is to use this questionnaire in other modalities of treatment such as surgery, radiotherapy, and supportive care. Thai FROM-16 may provide potential benefits from use in other diseases, such chronic kidney disease, neurological and cardiac disease. Further study for validation in those population, and questionnaire responsiveness may be required.

\section{Abbreviations}

CCRT: Concurrent Chemotherapy and radiation, ADL: activity of daily living; CFA: Confirmatory factor analysis; CFI: Comparative Fit Index; EFA: Exploratory factor analysis; FROM-16: Family Reported Outcome Measure 16; HRQOL: Health related quality of life HRQoL; NFI: Normed Fit Index; NNFI: Non-Normed Fit Index; RMSEA: Root Mean Square Error of Approximation; SRMR: Standardized Root Mean Square Residual; WHOQOLBREF-THAI: World Health Organization Quality of Life Brief-Thai WHOQOLBREF-THAI; X2: Chi Square test

\section{Acknowledgements}

We would like to thank nurses and all staff at Srinagarind hospital for their kindness and supports for this study.

The authors declare that they have no competing interests.

\section{Funding}

This study was partially funded by Faculty of Pharmaceutical Science, Khon Kaen University.

\section{Availability of data and materials}

Data sharing is available upon reasonable request. Kindly contact the corresponding author

\section{Authors' contributions}

CP performed literature review, data collection, statically analyses, and drafted the manuscript. JNP designed the protocol and revised the manuscript. PJ, SA, WK supervised and supported an enrollment. CA supervised the statistical analysis. MSS revised and finalized the manuscript. SS supervised the study planning and data collection. All authors reviewed and approved the final version of manuscript.

Ethics approval and consent to participate

The study protocol was approved by Khon Kaen University Ethics Committee in human research, record number 4.2.05:43/2015. Written informed consent was obtained from both family members and patients prior to data collection.

\section{Consent for publication}

Not applicable.

\section{Competing interests}

The authors declare that they have no competing interests.

\section{Publisher's Note}

Springer Nature remains neutral with regard to jurisdictional claims in published maps and institutional affiliations.

\section{Author details}

${ }^{1}$ The College of Pharmacotherapy of Thailand, Nonthaburi, Thailand. ${ }^{2}$ Faculty of Pharmaceutical Sciences and Melatonin Research Group, Khon Kaen University, Khon Kaen, Thailand. ${ }^{3}$ Palliative Care Unit, Faculty of Medicine, Khon Kaen University, Khon Kaen, Thailand. ${ }^{4}$ Medicine Unit, Faculty of Medicine, Khon Kaen University, Khon Kaen, Thailand. ${ }^{5}$ Division of Social and Administrative Pharmacy, Faculty of Pharmaceutical Sciences, Khon Kaen University, Khon Kaen, Thailand. 'SChool of Life \& Medical Sciences, University of Hertfordshire and Institute for Medicines Development, Hertfordshire, UK. ${ }^{7}$ Division of Clinical Pharmacy, Faculty of Pharmaceutical Sciences, Khon Kaen University, Khon Kaen, Thailand.

Received: 17 March 2018 Accepted: 14 January 2019

Published online: 08 February 2019

\section{References}

1. Wood-Dauphinee S. Assessing quality of life in clinical research: from where have we come and where are we going? J Clin Epidemiol. 1999:52(4):355-63.

2. Golics CJ, Basra MK, Finlay AY, Salek S. The impact of disease on family members: a critical aspect of medical care. J R Soc Med. 2013;106(10):399-407.

3. Covinsky KE, Goldman L, Cook EF, Oye R, Desbiens N, Reding D, et al. The impact of serious illness on patients' families. SUPPORT investigators. Study to understand prognoses and preferences for outcomes and risks of treatment. JAMA. 1994;272(23):1839-44.

4. Friethriksdottir $\mathrm{N}$, Saevarsdottir T, Halfdanardottir SI, Jonsdottir A, Magnusdottir $\mathrm{H}$, Olafsdottir $\mathrm{KL}$, et al. Family members of cancer patients: needs, quality of life and symptoms of anxiety and depression. Acta Oncologica (Stockholm, Sweden). 2011;50(2):252-8.

5. Mahrer-Imhof $R$, Jaggi $S$, Bonomo A, Hediger $H$, Eggenschwiler $P$, Kramer $G$, et al. Quality of life in adult patients with epilepsy and their family members. Seizure. 2013;22(2):128-35.

6. Edwards B, Clarke V. The psychological impact of a cancer diagnosis on families: the influence of family functioning and patients' illness characteristics on depression and anxiety. Psycho-Oncology. 2004;13(8):562-76. 
7. Varnum ME, Grossmann I, Kitayama S, Nisbett RE. The origin of cultural differences in cognition: evidence for the social orientation hypothesis. Curr Dir Psychol Sci. 2010;19(1):9-13.

8. Meecharoen W, Northouse LL, Sirapo-ngam Y, Monkong S. Family caregivers for Cancer patients in Thailand. SAGE Open. 2013;3(3):2158244013500280.

9. Golics CJ, Basra MK, Finlay AY, Salek S. The development and validation of the family reported outcome measure (FROM-16)(c) to assess the impact of disease on the partner or family member. Qual Life Res. 2014;23(1):317-26.

10. Vardiman JW, Thiele J, Arber DA, Brunning RD, Borowitz MJ, Porwit A, Harris NL, Le Beau MM, Hellström-Lindberg E, Tefferi A, Bloomfield CD. The 2008 revision of the World Health Organization (WHO) classification of myeloid neoplasms and acute leukemia: rationale and important changes. Blood. 2009;114(5):937-51.

11. Koller M, Aaronson NK, Blazeby J, Bottomley A, Dewolf L, Fayers $\mathrm{P}$, et al. Translation procedures for standardised quality of life questionnaires: the European Organisation for Research and Treatment of Cancer (EORTC) approach. Eur J Cancer. 2007:43(12):1810-20.

12. Mahatnirunkul S, Tuntipivatanakul W, Pumpisanchai W, et al. Comparison of the WHOQOL-100 and the WHOQOL-BREF (26 items). J Ment Health Thai. 1998;5:4-15.

13. Lim JW, Zebrack B. Caring for family members with chronic physical illness: a critical review of caregiver literature. Health Qual Life Outcomes. 2004;2:50.

14. Mukaka MM. Statistics corner: a guide to appropriate use of correlation coefficient in medical research. Malawi Med J. 2012:24(3):69-71.

15. Schumacker RE, Lomax, R.G. A beginner's guide to structural equation modeling. Second ed. New York: Routledge, Taylor \& Francis Group; 2010.

16. Cronbach $\sqcup$ J. Coefficient alpha and the internal structure of tests. Psychometrika. 1951;16(3):297-334

17. Tavakol M, Dennick R. Making sense of Cronbach's alpha. Int J Med Educ. 2011;2:53-5.

18. Kaiser HF. The application of electronic computers to factor analysis. Educ Psychol Meas. 1960;20(1):141-51.

19. Cattell RB. The scree test for the number of factors. Multivar Behav Res. 1966;1(2):245-76.

20. Hair JFTR, Anderson RE, Black W. Multivariate data analysis. fifth ed. London: Prentice-Hall; 1998

21. Ramada-Rodilla JM, Serra-Pujadas C, Delclos-Clanchet GL. Cross-cultural adaptation and health questionnaires validation: revision and methodological recommendations. Salud publica de Mexico. 2013;55(1): 57-66.

22. Kim SH, Jo MW, Lee SI. Psychometric properties of the Korean short form-36 health survey version 2 for assessing the general population. Asian Nurs Res. 2013;7(2):61-6.

23. Watcharee Leurmarnkul PM. Properties testing of the retranslated SF-36 (Thai version). Thai J Pharm Sci. 2005;29(1-2):69-88.

24. Mahendran R, Lim HA, Chua J, Peh CX, Lim SE, Kua EH. The caregiver quality of life index-Cancer (CQOLC) in Singapore: a new preliminary factor structure for caregivers of ambulatory patients with cancer. Qual Life Res. 2015;24(2):399-404.

25. Papadopoulos A, Vrettos I, Kamposioras K, Charitos D, Giannopoulos G, Pectasides $\mathrm{D}$, et al. Comparing health-related quality of life (HRQL) of cancer patients undergoing chemotherapy with family members in a tertiary hospital. J Clin Oncol. 2009 27:15S, e20535-e20535

Ready to submit your research? Choose BMC and benefit from:

- fast, convenient online submission

- thorough peer review by experienced researchers in your field

- rapid publication on acceptance

- support for research data, including large and complex data types

- gold Open Access which fosters wider collaboration and increased citations

- maximum visibility for your research: over $100 \mathrm{M}$ website views per year

At BMC, research is always in progress.

Learn more biomedcentral.com/submissions 